[Bull. Agr. Chem. Soc. Japan, Vol. 24, No. 7, p. 670 672, 1960]

\title{
Studies on the Components of the Hemp Plant (Cannabis sativa L.)
}

\author{
Part II. Isolation and Identification of piperidine and Several \\ Amino Acids in the Hemp Plant \\ By Yataro Obata, Yoshinori Ishikawa and Ryoichi Krtazawa \\ Department of Agricultural Chemistry, Faculty of Agriculture, Hokkaido University, Sapporo \\ Received June 4, 1960
}

\begin{abstract}
Investigation was undertaken to ascertain the components of the unpleasant odor of the hemp plant. The authors isolated and identified piperidine, as one of the unpleasant odor components of the plant, in ethanol soluble fraction of the plant.
\end{abstract}

\section{INTRODUCTION}

Almost all species of wild hemp exude a strong unpleasant odor in its flowering time and a lot of flies can be seen flying around the flower during this time.

Wild hemp used in this experiment was gathered right after the plant had flowered and was investigated for its unpleasant odor components.

In the previous paper the authors indicated that during the exploratory experiment on sulphur compounds in the process of beer brewing, humulone and lupulone in hop were found to be the cause of sunlight flavor of beer. ${ }^{1-6)}$ In this work the authors investigated the sulphur compounds in wild hemp to be helpful for elucidating the mechanism of sunlight flavor of beer because of hemp (Cannabis sativa L.) which in the same family (Moracea) with hop gives the similar unpleasant odor to the sunlight flavor of beer. Up to the present, free piperidine, combined piperidine and the following amino

\footnotetext{
1) Y. Obata and T, Yamanishi, Nogaku (Agriculture), 1, 537 (1947).

2) Y. Obota and T. Yamanishi, J.Agr. Cbem. Soc. Japan, 23, 154 (1949).

3) Y. Obata and T. Yamanishi, ibid., 24, 477 (1951).

4) Y. Obata and H. Horitsu, ibid., 32, 615 (1958).

5) Y. Obata and H. Horitsu, This Bulletin, 22, 153 (1958)

6) Y. Obata and H. Horitsu, ibid., 23, 186 (1959).
}

acids, leucine (or isoleucine), valine, methionine, threonine, histidine, arginine and lysine were isolated and identified from ethanol soluble fraction of the wild hemp.

\section{EXPERIMENTAL AND RESULTS}

\section{Sample:}

Hemp used in this experiment was one that grew wild in the fields of YUBARI COUNTY in HOKKAIDO.

Isolation and Identification of Piperidine by Paper Chromatography :

Leaves and tops of wild hemp $(10 \mathrm{~kg})$ were extracted with petroleum ether $\left(56 \mathrm{l}\right.$ ) (b.p. $28 \sim 42^{\circ} \mathrm{C}$ ) four times at room temperature and then the residue was extracted with $90 \%$ ethanol (50 1) three times at room temperature, this ethanol soluble fraction was concentrated in reduced pressure.

In the alcohol distillate, piperidine odor was detected and this ethanol distillate was adjusted to $\mathrm{pH} 3$ with hydrochloric acid and concentrated again to a small volume in vacuum.

When $30 \% \mathrm{KOH}$ (in water) was added to a portion of the concentrates, a strong unpleasant odor was detected, consequently the alkaline solution was extracted with ether and this ether extracted solution was dried with anhydrous sodium sulfate, and its concentrate was identified by paper chromatography using $n$-butanol-acetic acid-water $(4: 1: 2)$ at room temperature for twenty hours and followed by spraying with ninhydrin $(0.2 \%$ in ethanol) and exposing to vaporized iodine, both $R_{F}$ values $(0.54)$ of authentic piperidine and of separated 


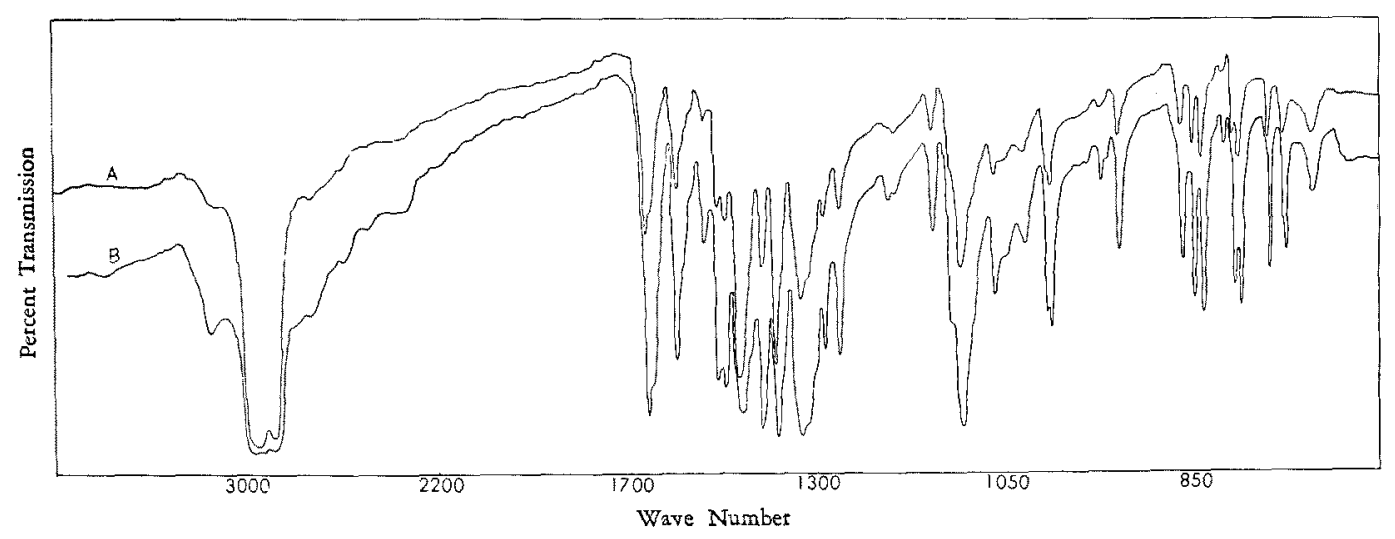

FIG. 1.

A: The infrared absorption spectrum of authentic piperidine picrolonate. (m.p. $242 \sim 244^{\circ} \mathrm{C}$ )

$\mathrm{B}$ : The infrared absorption spectrum obtained from hemp plant. (m.p. $242 \sim 245^{\circ} \mathrm{C}$ ) (Nujol).

\section{DIAGRAM}

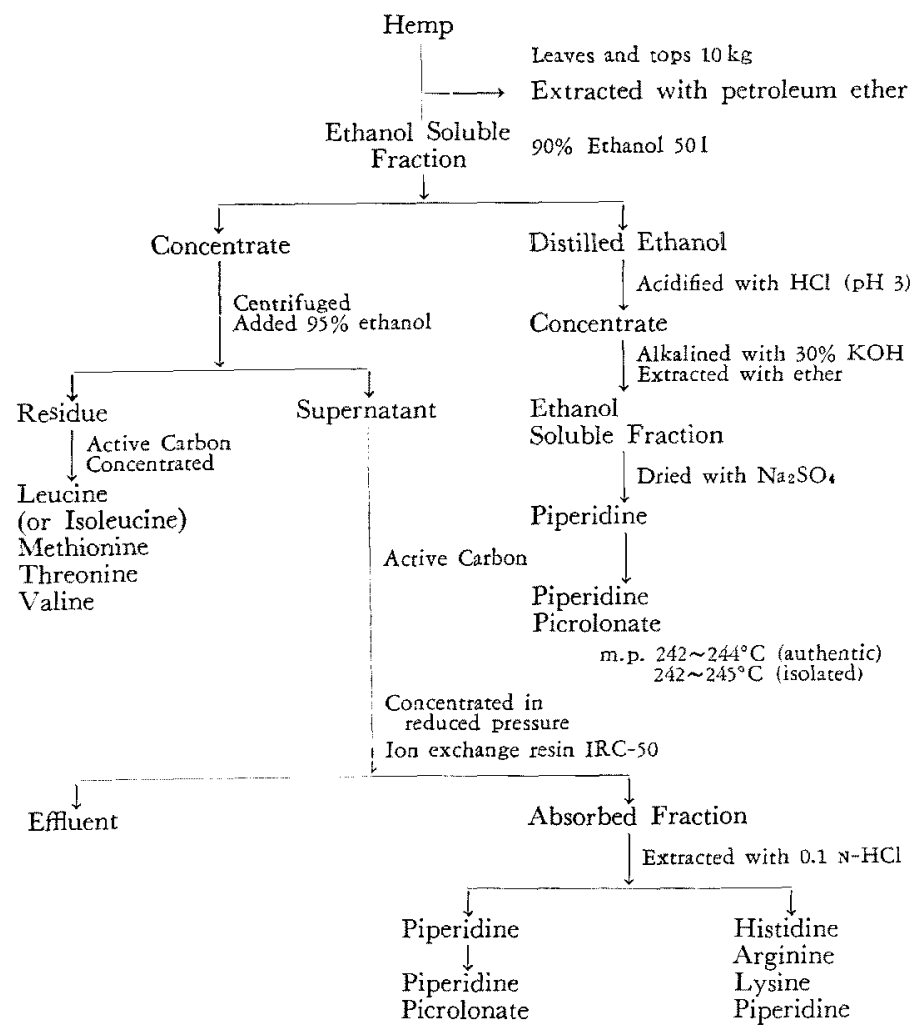


piperidine were found to be the same.

Preparation of Piperidine Picrolonate:

Piperidine picrolonate was prepared to identify the component detected by the chromatography as described above.

At first a portion of concentrates, acidified with hydrochloric acid, was placed in $100 \mathrm{ml}$ egg shaped flask and alkalined by adding $30 \% \mathrm{KOH}$. This flask was heated in the oil bath over $120^{\circ} \mathrm{G}$ for about 30 minutes and the evaporated free base was led to a test tube containing alcoholic solution saturated with picrolonic acid. This test tube was allowed to stand at room temperature over night. The red crystals of picrolonates obtained were filtered and dried up in a vacuum desiccator. The melting point of these crystals were measured by a micromelting point apparatus, comparing it with authentic piperidinc picrolonate, the former was $242 \sim 245^{\circ} \mathrm{C}$, the latter was $242 \sim 244^{\circ} \mathrm{C}$. The infrared spectra of these two derivatives in Nujol Mull were found to be coincident as shown Fig. 1 and Anal., found: $\mathrm{N}, 20.08$, calcd. for $\mathrm{C}_{15} \mathrm{H}_{19} \mathrm{~N}_{5} \mathrm{O}_{5}: \mathrm{N}, 20.06 \%$.

Isolation and Identificasion of Combined Piperidine and Several Amino Acids:

When 95\% ethanol was added to residue evaporated and cooled in refrigerator for a few days unknown crystals were obtained. These crystals were recrystalized several times from ethanol and filtered and chromatographed. The following amino acids; leucine (or isoleucine), valine, methionine, threonine were detected with ninhydrin $(0.2 \%$ in ethanol).

The mother liquid was treated with active carbon and then supernatant was passed through ion exchange resin IRC-50 and eluted with $0.1 \mathrm{~N}$-HCl. A portion of the elute was chromatographed after concetration to a small volume. Consequently piperidine was detected by the paper chromatography with spraying ninhydrin as well as arginine, histidine and lysine.

When $30 \% \mathrm{KOH}$ was added to the elute it gave off a strong piperidine odor, therefore, piperidine was isolated and identified by the same method of making derivatives of piperidine picrolonate as described above.

\section{SUMMARY}

In order to isolate and identify the unpleasant odor of wild hemp leaves, tops of the plant in its flowering time was extracted with petroleum ether at first and the residue was extracted with $90 \%$ ethanol. In this ethanol soluble fraction piperidine contained in free and combined form in hemp was isolated and identified by means of the paper chromatography analysis and infrared spectra.

The melting point of the picrolonate derived from wild hempen piperidine was $242 \sim 245^{\circ} \mathrm{C}$ and that of authentic piperidine was $242 \sim 244^{\circ} \mathrm{C}$. The infrared spectra of both picrolonates were found to be coincident and Anal., found: N, 20.08 , calcd. for $\mathrm{C}_{15} \mathrm{H}_{19} \mathrm{~N}_{5} \mathrm{O}_{5}: \mathrm{N}, 20.06 \%$.

Acknowledgements. The authors wish to express their thanks to Mr. T. Onishi, Department of Pharmacology, Hokkaido University for the infrared analysis. They are also greatly indebted to Dr. Y. Matsuoka, Eizai Co., Ltd., for the microanalysis 